\title{
Das transatlantische Handels- und Investitionsabkommen: Arbeitsplatzprognosen und Risikoanalysen
}

\author{
Nichtregierungsorganisationen, Gewerkschaften und viele mehr kritisieren das ange- \\ strebte Transatlantische Handels- und Investitionsabkommen (TTIP). Die Risiken, \\ die ein solches Abkommen birgt, betreffen Schutzstandards ebenso wie demokratische \\ Institutionen. Trotzdem soll es Studien zufolge den allgemeinen Wohlstand mehren. \\ Die Fallstricke und Grenzen, die mit den ökonomischen Prognosen verbunden sind, \\ nehmen ihrem Optimismus einiges an Strahlkraft. ${ }^{1}$
}

STEFAN BECK, CHRISTOPH SCHERRER

\section{Einleitung}

Die Europäische Kommission, Vertreter der Bundesregierung, wirtschaftsliberale Medien und Think Tanks werben seit Längerem für ein Transatlantisches Handels- und Investitionsabkommen (TTIP). Eine weitere Liberalisierung des transatlantischen Handels und die Erleichterung wechselseitiger Investitionen sollen durch den Abbau von Zöllen und „unnötigen“ Regulierungen den beteiligten Ländern aus der Krise helfen, ihre Ökonomien (bzw. Unternehmen) für den Wettbewerb mit den dynamisch aufstrebenden Ökonomien insbesondere des asiatischen Raumes stärken und neuen Schwung in die ins Stocken geratene multilaterale Liberalisierung des Welthandels bringen.

Tatsächlich geht es um weit mehr als nur kurz- oder mittelfristig realisierbare Wohlfahrtsgewinne aus einem intensivierten Handel. Vor dem Hintergrund der in jüngeren Handelsabkommen häufig vereinbarten und auch im TTIP beabsichtigten Sanktionsmöglichkeiten (v. a. für Investoren) und Festzurr-Effekte („lock-in“, z. B. Standstill-Klauseln, sprich Verbot neuer Regulierungen) geht es vielmehr um die langfristige gesellschaftliche, demokratische Gestaltbarkeit allokations-, verteilungs- und wohlfahrtsrelevanter Institutionen. Somit rücken neben den unmittelbaren wirtschaftlichen, ökologischen oder sozialen Effekten auch die langfristigen ökonomischen und politischen Verteilungswirkungen eines solchen Abkommens in den Vordergrund. Dies wiederum wirft die Frage nach den treibenden Interessen und Motivationen auf. Die medienwirksamen Streitpunkte z. B. über „Chlorhühner“, „Gen-Food“ oder „Fracking“ bilden nur die Spitze des Eisberges, um den die Verhandlungen kreisen. Darüber hinaus geht es um langfristige demokratische Gestaltungsmöglichkeiten und Verteilungsziele im nationalen und internationalen Raum. Es geht um die Steuerung öffentlicher Versorgungsdienstleistungen ebenso wie um die Möglichkeiten, sozial- oder umweltpolitische Ziele demokratisch zu bestimmen und zu verfolgen.

Im Folgenden werden wir zunächst überblicksartig auf wichtige Bereiche der TTIP-Verhandlungen eingehen und damit verbundene Risiken bzw. zentrale Kritikpunkte benennen (Abschnitt 2). Da die EU-Kommission und andere Freihandelsbefürworter demgegenüber das TTIP insbesondere mit Verweis auf signifikante Wohlfahrtsgewinne aufgrund zusätzlicher Exporte und Beschäftigungszuwächse rechtfertigen, gehen wir anschließend ausführlicher auf diese Argumente ein. Die Befürworter stützen sich auf ökonomische Studien, die auf Basis komplexer Modellrechnungen zu dem Ergebnis kommen, dass ein TTIP die Wohlfahrt in den Vereinigten Staaten (USA) und in der Europäischen Union (EU) steigern würde.

Dieser Artikel basiert auf einer ausführlicheren Studie zum TTIP, die von der Hans-Böckler-Stiftung gefördert wurde, vgl. Beck/Scherrer (2014). 
Während die meisten Studien auf computergestützten allgemeinen Gleichgewichtsmodellen beruhen (u. a. CEPR 2013), die mittlerweile in der Kritik stehen (vgl. Mitra-Kahn 2008; Taylor/von Arnim 2006), versuchen Georg Felbermayr et al. vom ifo Institut in München mithilfe eines alternativen Ansatzes, die Schwächen dieser Modelle zu überwinden. Da sich die ifo-Studien für das damalige Bundesministerium für Wirtschaft und Technologie (ifoBMWi, vgl. Felbermayr et al. 2013a) und für die Bertelsmann-Stiftung (ifo-Bertelsmann, vgl. Felbermayr et al. 2013b) zudem besonders mit den Konsequenzen für Deutschland befassen, konzentriert sich unser Artikel auf diese beiden Studien. Wir stellen zunächst die Ergebnisse dieser Studien vor (3). Dann setzen wir sie einer ausführlichen Kritik aus, prüfen ihre innere Konsistenz, fragen die Modellannahmen auf ihre Realitätsnähe ab und hinterfragen die unterstellten Kausalitäten (4). Das Ergebnis dieser Prüfung stellt nicht alle in diese Studien einfließenden analytischen und methodischen Überlegungen infrage. Wir kritisieren jedoch den Duktus, eine Fähigkeit zur sicheren Bestimmung der zukünftigen Wohlfahrtsgewinne eines Handelsabkommens zu behaupten (5).

\section{Risiken}

\subsection{Regulative und demokratische Risiken der Verhandlungen}

Anknüpfend an den bereits in den 1990er Jahren begonnenen, bislang aber wenig erfolgreichen transatlantischen $\mathrm{Di}$ alog soll es in den TTIP-Verhandlungen neben den verbliebenen - vergleichsweise geringen - Zöllen um einen umfassenden Abbau nicht-tarifärer Handelshemmnisse oder staatlicher Regulierungen bzw. um eine Anpassung regulativer und technischer Standards gehen. Ein solcher Abbau von Handelshemmnissen soll in horizontaler Hinsicht, d. h. sektorenübergreifend, und darüber hinaus sektorspezifisch vertiefend verhandelt werden. Dies kann auch umwelt-, gesundheits-, sozial- oder finanzpolitisch relevante Standards betreffen. Zwar beteuert die Europäische Kommission, dass es nicht um eine Absenkung solcher Standards gehen soll, entsprechende Befürchtungen sind jedoch durchaus begründet.

In demokratischer Hinsicht bedenklich ist nicht nur, dass vor allem große Unternehmen und deren Interessenverbände (z. B. Trans-Atlantic Business Council, European Services Forum) einen privilegierten Zugang zu den Verhandlungsführern genießen. Ebenso kritisch ist die freihändlerische Orientierung der verhandlungsführenden Vertreter, deren primäres Erfolgskriterium das Liberalisierungsziel und der Abbau „unnötiger" Regulierungen ist (vgl. Bartl/Fahey 2014). Sowohl die handelspolitische Tra- dition der EU als auch die Entwicklung des europäischen Binnenmarktes lassen befürchten, dass unter dem Primat der Marktöffnung Standards nach unten angepasst werden (vgl. De Ville/Orbie 2011; De Ville 2013).

Darüber hinaus sollen Liberalisierungs- und Deregulierungstendenzen dauerhaft festgeschrieben werden. Zum einen hat der Schwenk zu einem Negativlistenansatz eine expansive Wirkung, d. h. die Regulierung nicht explizit ausgenommener, vielleicht erst zukünftig relevanter Regelungsbereiche wird erheblich erschwert. Zum anderen sollen für bestimmte Bereiche oder Regelungen sogenannte Standstill-Klauseln vereinbart werden, die zukünftige ReRegulierungen untersagen. Und schließlich entfaltet das bislang angestrebte Investor-Staat-Streitschlichtungsverfahren wahrscheinlich verhindernde Wirkungen hinsichtlich demokratisch begründeter Regulierungen. Entsprechende Befürchtungen werden dadurch verstärkt, dass die Verhandlungen weitgehend geheim geführt werden und die Parlamente lediglich am Ende der Verhandlungen ein Mitbestimmungsrecht haben.

\subsection{Gefahren der Investor-Staat-Streitschlich- tung}

Das erstmals von der EU im Handelsabkommen mit Kanada (CETA) vereinbarte und ebenso im TTIP vorgesehene Investor-Staat-Streitschlichtungsverfahren (ISDS) im Bereich des Investitionsschutzes bildet zu Recht einen der zivilgesellschaftlich am heftigsten kritisierten Bestandteile der Verhandlungen. Im Rahmen eines ISDS haben Unternehmen die Möglichkeit, Staaten vor einem Schiedsgericht auf Entschädigung zu verklagen, wenn sie der Ansicht sind, dass staatliche Maßnahmen oder Regulierungen dem im Abkommen vereinbarten Investitionsschutz zuwiderlaufen.

Durch solche Streitschlichtungsverfahren werden nicht nur ausländische Investoren geschützt, sondern zugleich staatliche Regulierungsmöglichkeiten eingeschränkt, wenn diese nicht hohe Entschädigungszahlungen riskieren wollen. Dies beginnt mit unpräzisen Bestimmungen darüber, was ein legitimes öffentliches Interesse darstellt oder als eine indirekte Enteignung angesehen werden kann. Darüber hinaus handelt es sich um nicht-öffentliche, oft intransparente Verfahren, deren Schlichter oder Anwälte nicht hoheitlich legitimiert sind. Diese rekrutieren sich zumeist aus wenigen Kanzleien, die sich quasi abwechseln und die Rollen tauschen. Sie pflegen oft enge Beziehungen zu großen Unternehmen, prüfen laufend nationale Politiken und Regulierungsvorhaben auf Erfolg versprechende Klagemöglichkeiten, unterbreiten entsprechende Vorschläge und refinanzieren Klagen über den Finanzmarkt. Sowohl die hohen Honorare als auch das Interesse an einem Markt für Unternehmensklagen lassen vermuten, dass die Kanzleien hierbei zugleich Eigeninteressen verfolgen (Eberhardt 2013)

Aufgrund der oft hohen Streitwerte und Verfahrenskosten belasten solche Verfahren nicht nur die öffentlichen Haushalte, sondern stärken zugleich Investoren auf Kosten 
demokratischer Willensbildungsprozesse, wenn z. B. Regierungen aufgrund möglicher oder drohender Klagen von vorneherein auf Regelungen oder Politikmaßnahmen verzichten.

\subsection{Sektorspezifische und beschäftigungs- politische Risiken}

Das TTIP könnte auch erhebliche wettbewerbs- und beschäftigungspolitische Auswirkungen in verschiedenen Sektoren haben. Eine weitgehende Abschaffung von Zöllen oder Mengenbeschränkungen und die Angleichung von Verfahrensstandards im Agrarsektor würde die im Vergleich deutlich kleineren und produktionstechnisch weniger industriell ausgerichteten europäischen Betriebe erheblich belasten oder zum Teil gänzlich vom Markt verdrängen. Im Bereich der öffentlichen Beschaffung wiederum ist das europäische Interesse an einer Öffnung des US-Marktes größer als umgekehrt. Gleichwohl könnten die Absenkung der Schwellenwerte für Ausschreibungen auf das Niveau des EU-Binnenmarktes und eine Angleichung von Verfahrensstandards die öffentlichen Einflussmöglichkeiten hinsichtlich arbeitsoder umweltpolitischer Standards einschränken.

Auswirkungen eines transatlantischen Abkommens werden auch für den Dienstleistungshandel erwartet. Die Kommission ist bestrebt, den EU-Binnenmarkt für den Dienstleistungsbereich noch weiter zu liberalisieren, wobei das TTIP als Hebel dienen könnte. Im Einzelnen dürften die erwartbaren Folgen eines Abkommens je nach Dienstleistungsbereich und Erbringungsform variieren. Größere Handelseffekte werden in den Bereichen Informations-, Kommunikations-, Unternehmens- und Finanzdienstleistungen erwartet. Arbeitspolitische Auswirkungen, z. B. Absenkungen von Standards und erhöhter Lohndruck, sind darüber hinaus in beschäftigungsintensiven Bereichen wie Gesundheits- und Sozialen Dienstleistungen nicht auszuschließen.

Letzteres gilt ebenso für öffentliche (Versorgungs-) Dienstleistungen, zumal deren Definitionen nicht immer eindeutig sind und die EU-Kommission ein Interesse an einer möglichst weitgehenden Kommerzialisierung und strengeren wettbewerbspolitischen Regelungen hat. Im $\mathrm{Zu}$ sammenhang mit dem beabsichtigten Negativlistenansatz sind hier weitergehende Deregulierungen, Ausgliederungen oder Privatisierungen nicht auszuschließen.

\section{Die erwarteten Wohlfahrtseffekte der ifo-Studien}

Die Autoren der ifo-Studien haben sich viele Gedanken gemacht, wie die bisherigen Modellrechnungen zur Prognose der Auswirkungen von Handelsabkommen, von denen sie sich explizit abgrenzen, verbessert werden könnten. Die bisherigen Studien (z. B. CEPR 2013) treffen mehr oder weniger willkürliche Annahmen über das Ausmaß bestehender nicht-tarifärer Handelshemmnisse und darüber, in welchem Maße diese abgebaut werden können, d. h. in welchem Maße die Handelskosten durch ein Abkommen sinken. Die Autoren vom ifo-Institut hingegen versuchen, die Reduktion der Handelskosten implizit zu bestimmen. Hierzu schätzen sie zunächst mithilfe eines Gravitationsmodells ökonometrisch die Handelswirkungen früherer Abkommen. Ist demnach der zu erwartende Handelsschaffungseffekt - den die Autoren im Fall des TTIP auf nahezu $80 \%$ schätzen - bekannt, so lässt sich das Modell so kalibrieren, dass es exakt diesen Effekt simuliert. Darüber hinaus bemühen sie sich in einem weiteren Schritt um realistischere Annahmen bezüglich des Arbeitsmarktes und der Wettbewerbsbedingungen.

Die ifo-BMWi-Studie unterscheidet zunächst (in Kapitel 2) in einem 126 Länder umfassenden Modell zwischen einem Zollszenario, das lediglich einen Abbau der verbliebenen Zölle unterstellt, und einem Abkommen mit umfassender Liberalisierung, das den geschätzten Handelsschaffungseffekt vergangener Freihandelsabkommen zugrunde legt. Im Falle eines umfassenden Abkommens berechnen die Autoren einen Anstieg der deutschen Exporte in die USA um $94 \%$. Die Wohlfahrtseffekte schätzen die Autoren nach dem Konzept der äquivalenten Variation auf durchschnittlich rund $3 \%$ und knapp 4,7 \% für Deutschland in der langen Frist, welche die Autoren mit zehn bis 20 Jahren veranschlagen (Felbermayr et al. 2013a, S. 76). Für das Zollszenario hingegen fallen die Wohlfahrtsgewinne mit 0,24\% für Deutschland deutlich geringer aus (ebd., S. 81). Mittels der äquivalenten Variation werden im Durchschnitt $\mathrm{Zu}$ wächse in zehnfacher Höhe der Veränderungen des Bruttoinlandproduktes (BIP) gemessen, d. h. die Wohlfahrtseffekte erscheinen deutlich größer (Raza et al. 2014, S. 45f.).

In Kapitel 3 der ifo-BMWi-Studie entwickeln die Autoren dann ein modifiziertes Gleichgewichtsmodell (Übersicht 1). Dieses ist entsprechend dem zuvor ermittelten Handelsschaffungseffekt und der tatsächlichen bilateralen Handelsbilanzen (Gewichtung der Handelsbarrieren im Basisgleichgewicht) kalibriert, umfasst fünf aggregierte Regionen und wurde zur Ermittlung der Arbeitsmarkteffekte erweitert. Zu den Erweiterungen zählen insbesondere die Modellierung von Sucharbeitslosigkeit und Reallokationseffekten, die Berücksichtigung des Ausgangsniveaus der Arbeitslosigkeit (Daten: 2007) und die Annahme heterogener (Größe, Produktivität) Firmen (ebd., S. 83). Auf Basis dieses Modells werden nun drei Szenarien unterschieden: wiederum ein reines Zollszenario, ein NTB (Non-tariff barriers)-Szenario, das von einer zusätzlichen Absenkung nicht-tarifärer (ad valorem) Handelshemmnisse um $25 \%$ ausgeht und in etwa dem obigen Szenario einer umfassenden Liberalisierung entspricht, und ein Binnenmarktszenario, welches eine Absenkung aller effektiven bilateralen Handelshemmnisse auf das Niveau zwischen Deutschland und der EU unterstellt. Für das Zollszenario berechnen 
die Autoren eine Zunahme der deutschen Exporte in die USA um 4,86 \% und einen Rückgang der Exporte in die übrigen EU-Länder um 0,24 \%. Umgekehrt sollen die Exporte der USA nach Deutschland um 7,45 \% zunehmen, während die der übrigen EU um 0,44 \% schrumpfen. Im NTB-Szenario hingegen sollen die deutschen Exporte in die USA um knapp $57 \%$ zulegen und die in die übrige EU um 2,64 \% abnehmen. Die Exporte der USA nach Deutschland nehmen um knapp $94 \%$ zu und die der übrigen EU nach Deutschland um 5,5\% ab. Die im Gegensatz zur obigen Messgröße äquivalente Variation nun als BIP pro Kopf ausgewiesenen Einkommenszuwächse fallen geringer aus und belaufen sich im Zollszenario auf 0,13\%, im NTB-Szenario auf 1,6 \% und im Binnenmarktszenario auf $8,32 \%$ (ebd., S. $91 \mathrm{ff}$.).

Hinsichtlich des Arbeitsmarktes finden die Autoren für das Zollszenario keine nennenswerten Effekte auf das Beschäftigungs- und Lohnniveau. Dagegen sollen im NTBSzenario in Deutschland rund 25.000 neue Arbeitsplätze entstehen, in der übrigen EU fast 100.000 und in den USA

\section{ÜBERSICHT 1}

\section{Auswirkungen von TTIP auf Deutschland nach der ifo-BMWi-Studie}

Angaben in Prozent und in absoluten Zahlen

\begin{tabular}{lc|c|c}
\hline & Zollszenario & NTB-Szenario & $\begin{array}{c}\text { Binnenmarkt- } \\
\text { Szenario }\end{array}$ \\
\hline Export D -> USA & $+4,68 \%$ & $+57 \%$ & $+262 \%$ \\
\hline Export D -> EU26 & $-0,24 \%$ & $-2,64 \%$ & $-17,55 \%$ \\
\hline Reales BIP/Kopf & $+0,13 \%$ & $+1,6 \%$ & $+8,32 \%$ \\
\hline Reallöhne D & $+0,13 \%$ & $+1,6 \%$ & $+8,32 \%$ \\
\hline Arbeitsplätze D & +2.000 & +25.000 & +110.000 \\
\hline
\end{tabular}

Quelle: Felbermayr et al. (2013a), Kapitel 3.

Mitteilungen

ÜBERSICHT 2

\section{Auswirkungen von TTIP auf Deutschland nach der ifo-Bertelsmann-Studie}

Angaben in Prozent und in absoluten Zahlen

\begin{tabular}{lc|c}
\hline & Zollszenario & $\begin{array}{c}\text { Umfassende } \\
\text { Liberalisierung }\end{array}$ \\
\hline Export D -> USA & $+1,13 \%$ & $+94 \%$ \\
\hline Wohlfahrt D & $+0,24 \%$ & $+4,68 \%$ \\
\hline Reallöhne D & $+0,54 \%$ & $+2,19 \%$ \\
\hline Arbeitsplätze D & +45.000 & +181.000 \\
\hline
\end{tabular}

Quelle: Felbermayr et al. (2013b). knapp 70.000. Die durchschnittlichen Reallöhne sollen entsprechend jeweils um 1,6\%, knapp 1,7 \% und 2,15\% zulegen. Für das weitergehende Binnenmarktszenario (mit dem unrealistischerweise zum Teil geworben wird) ermitteln die ifo-Wissenschaftler sogar Beschäftigungsgewinne von bis zu 110.000 Arbeitsplätzen in Deutschland, 280.000 in der übrigen EU und 103.000 in den USA, wobei die Zuwächse vor allem in exportorientierten mittelständischen Unternehmen erfolgen sollen. Die durchschnittlichen Reallöhne wiederum sollen respektive um bis zu 8,32, 6,18 und $5,25 \%$ ansteigen. Die Beschäftigungs- und Reallohneffekte sollen dabei neben der Ausweitung des Handels insbesondere ein Resultat von Produktivitätssteigerungen und einer Absenkung des allgemeinen Preisniveaus sein. So soll die durchschnittliche Arbeitsproduktivität in Deutschland je nach Szenario (Zoll, NTB, Binnenmarkt) um 0,06, 1,14 bis hin zu 5,65\% ansteigen, während das Preisniveau um 0,06, 0,61 bzw. 16,19\% sinken soll (ebd., S. 97ff.).

Die ifo-Bertelsmann-Studie (Felbermayr et al. 2013b), die teilweise zu deutlich optimistischeren Schätzungen kommt, übernimmt zunächst die Methodik und Szenarien aus Kapitel 2 der ifo-BMWi-Studie, sodass die Handels- und Wohlfahrtseffekte mit diesen übereinstimmen (Übersicht 2). Im Zollszenario fallen die Exportzuwächse Deutschlands gegenüber den USA mit 1,13\% und die der USA gegenüber Deutschland mit 1,65 \% deutlich geringer als im kalibrierten Zollszenario in Kapitel 3/ifo-BMWi aus. Im Liberalisierungsszenario nehmen die Exporte nun wiederum in beide Richtungen um knapp 94 \% zu. Dagegen gehen die deutschen Ex- und Importe gegenüber den europäischen Ländern und der Mehrzahl der BRICS-Staaten mit bis zu 41 \% zum Teil deutlich zurück (ebd., S. 14ff.).

Bezüglich der Modellierung des Arbeitsmarktes weicht die Bertelsmann-Studie allerdings erheblich von der ifoBMWi-Studie $\mathrm{ab}$. Zum einen werden nicht wie oben in Übersicht 1 dargestellt neue umfassende Szenarien entwickelt, sondern lediglich die neu modellierten Arbeitsmarktdaten an die Szenarien ohne Arbeitsmarkt aus Kapitel 2/ ifo-BMWi angehängt. Und zum Zweiten unterscheidet sich die Modellierung des Arbeitsmarktes. Zwar wird ebenfalls Sucharbeitslosigkeit modelliert, jedoch nicht die Reallokation von Beschäftigung, d. h. der Beschäftigungsabbau in weniger produktiven Unternehmen bleibt unberücksichtigt. Zudem betrachtet die ifo-Bertelsmann-Studie nun 28 OECD-Länder und stützt sich auf Arbeitsmarktdaten aus den Jahren 2009 und 2010 (ebd., S. 32). Dementsprechend fallen bereits für das Zollszenario mit knapp 45.000 neu geschaffenen Arbeitsplätzen in Deutschland, 106.000 in Großbritannien, 277.000 in den USA und über 500.000 in der OECD die Zahlen deutlich optimistischer aus. Für das Liberalisierungsszenario nimmt die Studie sogar Beschäftigungszuwächse u. a. in Höhe von 181.000 Arbeitsplätzen in Deutschland und knapp 1,1 Mio. in den USA an. Dagegen sollen in anderen Ländern, wie z. B. Kanada, der Türkei oder Japan, jeweils über 50.000 und bis zu 100.000 Arbeitsplätze verloren gehen. 


\section{Kritik}

\subsection{Schwächen der ökonomischen Nutzen- kalkulationen}

Zweifellos versprechen ökonometrische Schätzungen eine bessere Abbildung der Realität, doch stellt sich die Frage, ob dieses Versprechen auch für einen Blick in die Zukunft eingelöst werden kann. Zunächst muss geklärt werden, inwiefern die bisherigen präferentiellen Handelsabkommen vergleichbar sind. Zum Vergleich zieht die Studie alle vor 2005 bei der WTO registrierten Abkommen heran, wobei weder das Alter noch die Qualität dieser Abkommen berücksichtigt werden. Viele dieser Abkommen sind jüngeren Datums. In welchem Zeitraum realisieren sich die erhofften Vorteile eines solchen Abkommens? Die Studie zielt explizit auf langfristige Effekte ab. Die lange Frist wird in der Studie dreimal spezifiziert, allerdings jedes Mal anders: fünf bis acht Quartale (Felbermayr et al. 2013a, S. 14 Fn13), zehn bis 20 Jahre (ebd., S. 69) und zwölf Jahre (ebd., S. 111). Je länger der Zeitraum, umso mehr nimmt die Wahrscheinlichkeit zu, dass die gemessenen Effekte durch andere Faktoren beeinflusst werden. Da die in der Studie herangezogenen Abkommen nicht nach dem Zeitverlauf bzw. Alter differenziert werden, ist deren Vergleichbarkeit fraglich. Und auch hinsichtlich der Qualität, sprich Liberalisierungstiefe, dieser Abkommen ist die Vergleichbarkeit nicht gegeben, da die Unterschiede allein zwischen den bekannten Abkommen, wie EU, NAFTA, Mercorsur und ASEAN, diesbezüglich erheblich sind. Ein Handelsabkommen von mit dem TTIP vergleichbarer Größe des Wirtschaftsraums besteht derzeit noch nicht. Insgesamt fehlt es für einen sinnvollen Vergleich hinsichtlich zentraler Parameter an Ähnlichkeit zwischen bestehenden Abkommen und dem TIPP.

Grundsätzlicher stellt sich aufgrund der negativen Erfahrungen mit Prognosemodellen auf Basis von Daten aus der Vergangenheit im Zuge der Finanzkrise (Taleb 2008) die Frage, ob überhaupt so umstandslos in der Vergangenheit gewonnene Daten in die Zukunft fortgeschrieben werden können. Gerade der technische Fortschritt erschwert den Blick in die Zukunft. So ermittelt die Studie beispielsweise, dass die US-Exporte von Erdöl und Erdgas nur leicht steigen sollen (Felbermayr et al. 2013a, S. 115). Angesichts der Erschließung von Schieferöl- und -gasvorkommen durch die neuen Fracking-Techniken wird jedoch derzeit ein anderes Volumen prognostiziert (US-Department of Energy 2009). Zum Abschluss des Dienstleistungsabkommens GATS im Jahre 1994 konnten sich die beteiligten Bildungsministerien noch nicht vorstellen (Scherrer 2005), dass mittels des Internets Universitäten wie die Phoenix University grenzüberschreitend Hunderttausende von Studierenden mit Kursen versorgen können.

Bei der Bemessung der wirtschaftlichen Auswirkungen von präferentiellen Handelsabkommen stellen Faktoren wie
Technik auch für den Blick in die Vergangenheit eine Herausforderung dar. Wie kann der Anteil eines Handelsabkommens an der generellen Vertiefung grenzüberschreitender Arbeitsteilung gegenüber anderen Faktoren genau bemessen werden? Und noch grundsätzlicher: Kann die Wirkung eines einzelnen Faktors in komplexen Systemen wie menschlichen Gesellschaften überhaupt isoliert werden? Dies würde voraussetzen, dass die Wirkung des untersuchten Faktors unabhängig von der jeweiligen spezifischen Interaktion mit anderen Faktoren ist. Ein Abkommen über grenzüberschreitende Energiemärkte wirkt z. B. vor dem Einsatz von „Fracking“ anders als danach. Mit anderen Worten: Die jeweils gemessene Wirkung kann Produkt des $\mathrm{Zu}$ sammenspiels verschiedener Faktoren sein (Ragin 2008).

Die ifo-Studie geht allerdings davon aus, dass der Faktor Handelsabkommen in seiner Wirkung isoliert werden kann, indem neben der Existenz eines solchen Abkommens auch der Einfluss weiterer Variablen, wie z. B. die räumliche Nähe, eine gemeinsame Grenze oder Sprache auf die Handelsbeziehungen geschätzt werden, wobei letztgenannte Faktoren selbst wiederum Einfluss auf die Wahrscheinlichkeit, dass es zu einem Abkommen kommt, haben können. Damit soll der Ansatz in der Lage sein, sowohl die Handelsschaffungsals auch die Handelsumlenkungseffekte (weg von den Partnern außerhalb, hin zu denen innerhalb der Freihandelszone) zu berücksichtigen (Felbermayr et al. 2013a, S. 59).

Da präferentielle Handelsabkommen über den Abbau von Zöllen hinausgehen, stellt sich bei der Ermittlung der Auswirkungen das Problem, wie die nicht-tarifären Handelsbarrieren (NTB) gemessen werden können. Für ihre Analyse auf nationaler Ebene (Makroanalyse) meint die ifo-Studie, das Problem mit Hilfe des Gravitationsmodells elegant umgehen zu können. Es werden einfach die handelsschaffenden Faktoren in ihrer Gesamtheit gemessen, da es letztlich offen bliebe, ob die empirisch gemessenen Handelseffekte aus der Reduktion tarifärer oder dem Abbau nicht-tarifärer Barrieren stammen. Ob aber mittels der Gravitationsgleichung ohne Spezifizierung der Handelsabkommen nach Dauer und Tiefe und ohne Berücksichtigung von Interaktionseffekten dies mit der Genauigkeit gelingt, die die Studie vorgibt zu erreichen, ist zweifelhaft. Gerade wenn zeitliche Effekte untersucht werden sollen, bietet sich die Längsschnittanalyse an, die aber hier wohl aus Gründen der schwierigen Datenerhebung nicht erfolgte. Zudem bleiben die Fragen sowohl nach der Vergleichbarkeit für das TTIP als auch der Tauglichkeit vergangener Daten für Prognosen offen.

\subsection{Bemessung der nicht-tarifären Handelsbarrieren}

Während für die Makroanalyse die ifo-BMWi-Studie auf eine exakte Bemessung der Höhe der NTB verzichtet, versucht sie genau dies für ihre sektoralen Analysen. Da es derzeit allerdings keine anerkannte Methodik gebe, mit deren Hilfe die Kosten von nichttarifären Handelsbarrieren berechnet werden könnten (Felbermayr et al. 2013a, 
S. 42), geht die Studie zweigleisig vor. Zum einen setzt sie die für die Makroanalyse abgelehnten Berechnungsmodelle in leicht modifizierter Weise ein. Weil sie die so ermittelten Ergebnisse bloß „als informativ, aber nur teilweise belastbar" (ebd., S. 43) ansieht, ergänzt sie diese Analysen innovativ mit den Resultaten einer Umfrage unter deutschen Wirtschaftsverbänden zu den Handelskosten in den USA. Eine Befragung US-amerikanischer Verbände wurde nicht vorgenommen, sodass für die Handelskosten in Europa keine Umfrageergebnisse vorliegen. Im Fließtext der Studie liest es sich zunächst so, als ob die Rücklaufrate sehr hoch sei (ebd., S. 48), doch ein Blick auf die komplette Liste der Antworten in Tabelle A. I.1 zeigt, dass nur 16 Verbände geantwortet haben, wovon auch etliche die Umfrage nicht vollständig beantworteten. Die Antworten stammen eher von Verbänden, die sich schon zuvor für das TTIP eingesetzt haben. Gleichwohl sind einige Antworten durchaus interessant. So wurden allgemein als Barrieren die Gesetze zur Terrorismusbekämpfung in den USA genannt. Inwiefern diese aufgrund des TTIP geändert werden, bleibt unklar. Des Weiteren wurden insbesondere sektorale Hürden benannt. Für die Finanzindustrie ist dies das komplexe Gesetzeswerk in Reaktion auf die Finanzkrise von 2007, der DoddFrank Act. Für die Nahrungsmittelindustrie sind dies die besonderen Veterinär- und Hygieneregelungen. Die Studie wirft nicht die Frage auf, ob solche Regelungen sinnvoll sind und deshalb nicht durch das TTIP beseitigt werden sollten.

Durch die Umfrage fühlt sich die Studie darin bestätigt, die Berechnungen rein auf einer Güterwirtschaft fußen und monetäre Aspekte außen vor lassen zu können, da „die Wechselkursproblematik [...] außer im Textilsektor und im Fahrzeugbau von den Interviewpartnern als wenig wichtig eingestuft" (Felbermayr et al. 2013a, S. 55) wird. Allerdings sieht die Studie an anderer Stelle gerade diese zwei Sektoren als diejenigen an, die am meisten vom TTIP profitieren würden (ebd., S.18). Wechselkursschwankungen übertreffen die bestehenden Zollsätze bei Weitem; zwischen 2002 und 2008 stieg der Euro gegenüber dem US-Dollar um $64 \%$ (Priewe 2013, S. 126).

Bei der ökonometrischen Analyse der nicht-tarifären Handelsbarrieren zwischen der EU und den USA geht die Studie davon aus, dass innerhalb der USA, der nordamerikanischen Freihandelszone (NAFTA) und insbesondere innerhalb der EU keine Handelsbarrieren bestehen. Laut der Studie macht diese Annahme "durchaus Sinn" (Felbermayr et al. 2013a, S. 88 Fn. 47). Selbst innerhalb der USA bestehen jedoch gerade im Dienstleistungssektor Handelsbarrieren, beispielsweise bei der gegenseitigen Anerkennung von professionellen Zertifikaten. Einzelstaatliche Regelungen für den Umweltschutz führen auch im Gütersektor zu Barrieren, beispielsweise die unterschiedlichen Abgasnormen für Automobile. Die Nichtberücksichtigung der internen Barrieren erhöht die vermuteten externen Barrieren. So errechnet die Studie, dass die „imputierten“ (sic!), d. h. geschätzten, Handelskosten für Exporte Deutschlands in die USA einen Wert von 53 annehmen, sprich $53 \%$ teurer sind als in Deutschland (Felbermayr et al. 2013a, S. 89). US-Exporte nach Deutschland sind sogar $155 \%$ teurer als in den USA. Die hohe Differenz erklärt sich „vor allem damit [...], dass Deutschland im Jahr 2007 hohe bilaterale Überschüsse erwirtschaftet hat" (ebd., S. 89) und ist ein Resultat der Kalibrierung des Modells. Die Exportüberschüsse weisen demnach auf Barrieren für die Importe hin, denn ohne die Barrieren müssten nach der Gleichgewichtslogik der Studie Ex- und Importe ausgeglichen sein. Da die Überschüsse Deutschlands in der Folgezeit noch stärker gewachsen sind, müssten folglich auch die Handelsbarrieren nach Deutschland zugenommen haben. Wird somit die Höhe der Handelsbarrieren als eine Funktion von Außenhandelsbilanzungleichgewichten gemessen, ändern sich die Handelsbarrieren von Jahr zu Jahr, je nach Stand der Außenhandelsbilanz. Dies ist jedoch wenig plausibel.

\subsection{Beschäftigungseffekte}

Wie oben erwähnt, versucht die Studie in die Berechnungsmodelle der Auswirkungen von Handelsabkommen möglichst realistische Annahmen einzuführen, wie z. B. die Berücksichtigung des vorherrschenden Beschäftigungsniveaus und bestehender Arbeitsmarktinstitutionen (ebd., S. 84). Die Studie wählt das Jahr 2007 als Basisszenario, ein Jahr mit vergleichsweise hoher Arbeitslosigkeitsrate in Deutschland. Wäre das Jahr 2011 gewählt worden, würden sich die Beschäftigungseffekte nach Modellrechnung wohl unterschiedlich auf die Handelspartner verteilen, sprich die deutschen Zugewinne geringer sein. Statt eines Stichjahres wäre es sicherlich angemessener, das durchschnittliche $\mathrm{Ni}$ veau eines Konjunkturzyklus als Ausgangspunkt zu wählen. Allerdings stellt sich auch dann die Frage, ob die jeweiligen Arbeitslosen tatsächlich das Qualifikationsprofil mitbringen, das in den wachsenden Exportindustrien gesucht wird.

Darüber hinaus bleibt die Modellierung des Anpassungsprozesses unbefriedigend. Empirische Studien zu den Suchprozessen von Arbeitskräften, die durch die internationale Konkurrenz oder technologische Fortschritte freigesetzt wurden, zeigen sowohl für die USA als auch für Deutschland für einen signifikanten Teil der Arbeitslosen recht lange Suchzeiten auf und zumeist Lohneinbußen in der neuen Arbeitsstelle (Scott 2012; zur Bewertung des NAFTA-Abkommens: Public Citizen 2010). Ebenso wenig ersichtlich ist die konkrete Zusammensetzung des Indikators „Matching Effizienz” (Felbermayr et al. 2013a, Tab. III.2), der die Leistungsfähigkeit der Arbeitsmarktinstitutionen bei der Zusammenführung von Angebot und Nachfrage abbilden soll. Welche Institutionen besonders leistungsfähig sind, ist in der Literatur sehr umstritten (Bhaumik/Dimova 2014).

Die Arbeitsplatzgewinne werden für drei Szenarien berechnet, wobei in dem von der Studie präferierten zweiten Szenario (NTB-Szenario) die Handelsbarrieren nicht völlig verschwinden, sondern nur vom jeweiligen Niveau um 25 Prozentpunkte abgesenkt werden (Felbermayr et al. 2013a, 
S. 90). In diesem „realistischen“ "Szenario würden in Deutschland 25.000 neue Arbeitsplätze entstehen (ebd., S. 100), was bei einer Erwerbstätigenzahl von 41,8 Mio. (2012) etwa ein halbes Promille ausmacht. Diese neuen Arbeitsplätze sollen zudem sicherer sein: ,außerdem gelingt die Umwandlung von in der Regel unsicheren Arbeitsplätzen in Einmann- und Kleinstunternehmen zu Jobs in mittelständischen Firmen" (ebd., S. 102). Insgesamt sollen in der neu geschaffenen Freihandelszone 193.000 zusätzliche Arbeitsplätze entstehen, allerdings auf Kosten der Länder außerhalb des TTIP, welche 165.000 Arbeitsplätze verlieren würden (ebd., S. 100).

Die ifo-Bertelsmann-Studie kommt zu deutlich höheren Gewinnen (Felbermayr et al. 2013b, s. Tabelle 2). Dies ist Folge eines geringeren Aggregationsniveaus (statt Weltregionen 28 OECD-Länder), der Vernachlässigung von Reallokationseffekten und von Annahmen über Produktivitätseffekte: Durch die Handelsliberalisierung freigesetzte Arbeitskräfte wechseln nicht wie in der ifo-BMWi-Studie in produktivere Unternehmen, was die Beschäftigungseffekte mindern würde, sondern in weniger produktive Branchen. Welche Annahmen zutreffen, können wir nicht beurteilen. Wir stellen wiederum fest, dass die Ergebnisse der Studien stark von ihren Annahmen beeinflusst werden, ohne dass dies in den Studien problematisiert wird.

Die handelsinduzierten Produktivitätszuwächse würden zudem höhere Löhne ermöglichen, in Deutschland um die $50 €$ höhere durchschnittliche Bruttomonatsverdienste (Felbermayr et al. 2013a, S. 103). Diese Lohnsteigerungen seien „sehr stark vom Preisindex getrieben” (ebd., S. 104). Dieser wiederum von handelsgetriebenen Produktivitätsentwicklungen, die „zu einer Reduktion der durchschnittlichen Preise für heimische Konsumenten führen" (ebd., S. 99). Die eingangs erwähnte Berücksichtigung von Marktunvollkommenheiten findet hier keinen Niederschlag. An dieser Stelle nimmt die Studie an, dass die Produktivitätszuwächse aufgrund der Konkurrenzverhältnisse direkt an die Konsumenten weitergegeben werden. Die Auswirkungen von Währungsschwankungen auf den Preisindex werden ebenfalls nicht berücksichtigt.

In üblicher neoklassischer Argumentation wird vor diesen Lohnsteigerungen gewarnt: „Je stärker die Reallöhne steigen, umso weniger kann die Beschäftigung steigen" (ebd., S. 86), doch glücklicherweise „lässt es sich im Rahmen des Modells zeigen, dass Handelsliberalisierung nicht $\mathrm{zu}$ einer Abnahme der Beschäftigung führen kann: im langfristigen Gleichgewicht überwiegt die Schaffung von Arbeitsplätzen in exportorientierten Firmen die Arbeitsplatzverluste in nicht exportorientierten Firmen" (ebd., S. 86, Hervorhebung der Autoren). Das Modellergebnis wird hier wiederum für bare Münze genommen.

\subsection{Handelsumlenkung}

Dem Vorwurf, dass ein bilaterales Handelsabkommen wie das TTIP den Anspruch der Welthandelsorganisation auf multilaterale, sprich alle Handelsnationen einschließende
Abkommen untergräbt (Langhammer 2008), begegnet die ifo-BMWi-Studie mit dem Argument, dass in den letzten Jahrzehnten solche bilateralen Abkommen nicht weitere multilaterale Abkommen verhindert hätten. Im Gegenteil, oftmals hätten Erstere als Trittbrett für weitere Handelsliberalisierungen auf multilateraler Ebene fungiert. Die Ausdehnung der im TTIP vereinbarten Liberalisierungen auf andere Handelspartner ist auch erklärtes Ziel der EU-Kommission und der US-amerikanischen Regierung, die beide über die geringen Fortschritte innerhalb der Welthandelsorganisation klagen. Die durchaus problematische machtpolitische Dimension dieser Ausdehnung wird von der Studie nicht thematisiert, was allerdings nicht verwundert, da ihr Grundtenor vom Freihandelsparadigma geprägt ist.

Beunruhigend sind allerdings die Ergebnisse der Studie hinsichtlich der zu erwartenden Handelsumlenkungen durch das TTIP, bevor es zu seiner Verallgemeinerung im Rahmen der World Trade Organization (WTO) kommt. Bei einer Mehrzahl der deutschen Handelspartner wird ein Rückgang des Handelsvolumens erwartet. Laut der Studie darf daraus „aber noch nicht geschlossen werden, “ dass das TTIP insgesamt für Deutschlands Handelspartner „keine positive Wirkung hat" (Felbermayr et al. 2013a, S. 75), denn die Reduzierung des Handels mit einem Nicht-TTIP-Land zugunsten des Handels mit einem TTIP-Land könnte aufgrund geringerer Handelskosten zu einem höheren BIP führen (Felbermayr et al. 2013a, S. 75). Allerdings widersprechen die von der Studie gemessenen Wohlfahrtseffekte dieser Aussage. Beim umfassenden Szenario kommt die Studie auf erhebliche Wohlfahrtsverluste für Japan (-5,9\%), Mexiko (-7,24 \%), Australien (-7,41 \%) und Kanada (-9,48 \%). Diesen sollen Zugewinne in den USA von $13,38 \%$, in Großbritannien von 9,7 \%, in Schweden von $7,3 \%$ und in Spanien von 6,55 \% gegenüberstehen (ebd., S. 76 Abb. II.1). Die Verlierer sind vornehmlich die Länder, die mit den USA oder der Europäischen Union bereits Freihandelsabkommen unterhalten. Beim Zollszenario fallen Gewinne und Verluste deutlich geringer aus, doch der Verlust für Indien in Höhe von -2,48 \% fällt deutlich höher aus als der Zugewinn für den größten Gewinner, die USA mit $+0,75 \%$ (ebd., S. 81 Abb. II.2). Innerhalb der EU rechnet die ifo-Bertelsmann-Studie mit erheblichen Einbrüchen im Handelsvolumen, z. B. zwischen Deutschland und Irland um 35 \% (Felbermayr et al. 2013b, S. 15), und bei den Handelspartnern außerhalb des TTIP ebenso (z. B. Deutschland - China um 13 \% [ebd., S. 16]) - als Spiegelbild der höheren handelsschaffenden Effekte innerhalb des TTIP. Zudem könnten die Umlenkungseffekte noch unterschätzt sein, da in der ifo-BMWi-Studie Ursprungsregelungen im Fall eines Abbaus nicht-tarifärer Hemmnisse als unbedeutend betrachtet werden (Felbermayr et al. 2013a, S. 18). Werden jedoch statt tatsächlicher Harmonisierungen Formen wechselseitiger Anerkennung zwischen den beteiligten Ländern vereinbart, so müssen Drittländer nach wie vor unterschiedlichen Standards folgen und haben dadurch einen Wettbewerbsnachteil (vgl. Bartl/Fahey 2014). 
Sollten sich diese Zahlen als korrekt erweisen, so wäre mit erheblichen außenpolitischen Spannungen zu rechnen. Zudem kommt es in den Worten vom Befürworter eines weltweiten Freihandels, Jagdish Bhagwati, zu einer „Spaghetti bowl“ (1995) von unterschiedlichen Rechtsnormen und Rechtsprechungen. Dies sind wiederum weitere Dimensionen, zu der die Studie, aber auch die politischen Betreiber des TTIP schweigen.

\section{Fazit}

Angesichts der erwartbaren oder nicht auszuschließenden demokratischen, regulativen, sozial-, umwelt- und gesundheitspolitischen Risiken eines transatlantischen Handels- und Investitionsabkommens lohnt sich ein genauerer Blick auf die versprochenen Wohlfahrtsgewinne. Die genaue Betrachtung der ifo-Studien zu den Wohlfahrtseffekten eines solchen Abkommens weckt Zweifel an der Gewissheit ihrer Prognosen. Zum einen vernachlässigen sie mögliche positive Auswirkungen von wirtschaftlichen Regulierungen sowie die kurz- oder mittelfristigen negativen Effekte auf den Absatz und die Beschäftigung in Wirtschaftszweigen, die durch Handelsumlenkungen, vor allem innerhalb der EU, oder erhöhte Importkonkurrenz betroffen sind. Zum anderen ist fraglich, ob es diesen Studien tatsächlich gelingt, durch ökonometrische Schätzungen der Modellparameter und durch die Berücksichtigung von Arbeitslosigkeit sowie imperfekten Wettbewerb die bekannten Schwächen der rechenbaren allgemeinen Gleichgewichtsmodelle zu überwinden.

Die vorgenommenen ökonometrischen Querschnittsanalysen mittels der Gravitationsgleichung ohne Spezifizierung der Handelsabkommen nach Dauer und Tiefe und ohne Berücksichtigung von Interaktionseffekten lassen an der Genauigkeit, die die Studie vorgibt zu erreichen, zweifeln. Zudem bleiben die Fragen sowohl nach der Vergleichbarkeit für das TTIP als auch der Tauglichkeit vergangener Daten für Prognosen offen. Die Berücksichtigung der Arbeitslosigkeit erfolgt im Rahmen neoklassischer Denkschablonen, die bekanntermaßen vorurteilsgeladen die Wirkung von Arbeitsmarktinstitutionen auf ein simples Schema von Angebot und Nachfrage reduzieren. Die Berücksichtigung von eingeschränkter Konkurrenz blieb in den Studien ein uneingelöstes Versprechen.

Abschließend soll noch einmal betont werden, dass diese ifo-Studien tatsächlich innovativ sind und grundsätzlich einen Fortschritt gegenüber den bisherigen rechenbaren Allgemeinen Gleichgewichtsmodellen darstellen. Irritierend ist allerdings, dass die errechneten Ergebnisse als objektive Fakten präsentiert werden. Obgleich die prognostizierten positiven Auswirkungen deutlich über denen anderer Studien liegen, sollen alle Ergebnisse sogar bloß ,als Untergrenzen für langfristige Effekte” (Felbermayr 2013a, S. 14) verstanden werden. Bei der ifo-Bertelsmann-Studie heißt es zum Beispiel „Wir vergleichen also die faktische, wirklich beobachtete Realität ..." (ebd., S. 13), wobei allerdings die verwendete Gravitationsgleichung eine lineare Projektion darstellt, die die „Realität“ von Zufallseinflüssen $\mathrm{zu}$ bereinigen versucht. Interessanterweise taucht das Wort „Prognose“ in der Studie nicht auf. Wie üblich für wissenschaftliche Prognosen wird zwar mit Szenarien gearbeitet, doch diese beziehen sich lediglich auf das vermutete Ausmaß an Liberalisierung durch das TTIP. Szenarien auf der Basis unterschiedlicher Modellannahmen fehlen. Für jede Stufe an Liberalisierung wird jeweils nur ein Auswirkungsszenario vorge- stellt, obgleich obige Diskussion die großen Ermessensspielräume hinsichtlich zentraler Modellannahmen aufzeigt. Dass die Auftraggeber dieser Studien, die sich bereits zuvor zugunsten des TTIP festgelegt hatten, mögliche vorsichtige Einschränkungen einer wissenschaftlichen Studie geflissentlich übersehen, ist wenig verwunderlich. Leider haben diese ifo-Studien ein solches Verhalten besonders leicht gemacht.

\section{LITERATUR}

Bartl, M./Fahey, E. (2014): The postnational market place: Negotiating the Transantlantic Trade and Investment Partnership (TTIP), in: Curtin, D./Fahey E. (Hrsg.): A transatlantic community of law: Legal perspectives on the relationship between the EU and US legal orders, Cambridge, S. 210-234

Beck, S./Scherrer, C. (2014): Das transatlantische Handels- und Investitionsabkommen (TTIP) zwischen der EU und den USA: Hans-Böckler-Stiftung, Arbeits papier, Globalisierung und Europäisierung (303), Düsseldorf

Bhagwati, J. (1995): U.S. trade policy: The infatuation with free trade areas, in: Bhagwati, J./Krueger, A. O. (Hrsg.): The dangerous drift to preferential trade agreements, Washington DC, S. 1-18

Bhaumik, S./Dimova, R. (2014): Good and bad institutions: Is the debate over? Cross-country firm level evidence from the textile industry, in: Cambridge Journal of Economics 38 (1), S. 1-21

Centre for Economic Policy Research (CEPR) (2013): Reducing transatlantic barriers to trade and investment. An economic assessment, Final Project Report, London, http://trade.ec.europa.eu/doclib/docs/2013/march/tradoc_ 150737.pdf

De Ville, F. (2013): EU trade policy as the continuation of internal market policy by other means, in:Takács, T./Ott, A./Dimopoulos A. (Hrsg.): Linking trade and non-commercial interests: The EU as a global role model?: Center for the Law of EU External Relations (CLEER), Working Papers 2013/4, S. 93-103

De Ville, F./Orbie, J. (2011): The European Union's trade policy response to the crisis: Paradigm lost or reinforced?, European Integration online Papers 15 (2) http://eiop.or.at/eiop/texte/2011-002a.htm

Eberhardt, P. (2013): Politikbekämpfung als Geschäftsmodell. Die Rolle der Rechtsbranche im internationalen Investitionsrecht, in: juridikum 3/2013, S. 386-391

Egger, P./Larch, M./Staub K. E./Winkelmann R. (2011): The trade effects of endogenous preferential trade agreements: Dataset, in: American Economic Journal, Economic Policy 3 (3), S. 113-143, http://www.aeaweb.org/articles. php?doi=10.1257/pol.3.3.113.

Felbermayr, G./Larch, M./Flach, L./Yalcin, E./Benz, S. (2013a): Dimensionen und Auswirkungen eines Freihandelsabkommens zwischen der EU und den USA. Studie im Auftrag des Bundesministeriums für Wirtschaft und Technologie, München

Felbermayr, G./Heid, B./Lehwald, S. (2013b): DieTransatlantische Handels- und Investitionspartnerschaft (THIP). Wem nutzt ein transatlantisches Freihandelsabkommen? Teil 1: Makroökonomische Effekte, Gütersloh

Langhammer, R. (2008): Why a market place must not discriminate: The case against a US-EU free trade agreement: Kiel Institute for the World Economy, Working Paper (1407)

Mitra-Kahn, B. H. (2008): Debunking the myth of general equilibrium models: Schwartz Center for Economic Policy Analysis, The New School for Social Research, SCEPA Working Paper 2008-1, New York

Priewe, J. (2013): Transatlantic trade partnership versus transatlantic currency cooperation, in: Palley, Th./Horn G. (Hrsg.): Restoring shared prosperity, LCCN 2013923093, S. 123-132

Public Citizen (2010): Lies, damn lies and export statistics. Public Citizen's global trade watch, Washington DC, http://www.citizen.org/documents/ FTA\%20 Penalty\%20Paper\%20FINAL1.pdf

Ragin, C. C. (2008): Redesigning social inquiry: Fuzzy sets and beyond, Chicago 
Raza, W./Grumiller, J./Taylor, L./Tröster, B./Arnim, R. v. (2014): Assess_TTIP: Assessing the claimed benefits of the Transatlantic Trade and Investment Partnership: ÖFSE - Austrian Foundation for Development Research, Wien Scherrer, C. (2005): The Role of GATS in the commodification of education; in: Hamm, B./Smandrych, R. (Hrsg.): Cultural imperialism. Essays on the political economy of cultural domination, Peterborough, S. 167-190

Scott, R. E. (2012): The China toll: Economic Policy Institute, EPI Briefing Paper (345), Washington DC

Taleb, N. N. (2008): Der schwarze Schwan: Die Macht höchst unwahrscheinlicher Ereignisse, München

Taylor, L./Arnim, R. v. (2006): Modelling the impact of trade liberalisation. A critique of computable general equilibrium models, Oxfam International Research Report, http://oxfamilibrary.openrepository.com/oxfam/bitstream/ 10546/112547/1/rr-modelling-impact-trade-liberalisation-010707-en.pdf US Department of Energy (2009): Modern shale gas development in the United States: A primer, Washington DC, http://www.netl.doe.gov/ technologies/oil-gas/publications/epreports/shale_gas_primer_2009.pdf

\section{AUTOREN}

STEFAN BECK, Dr., ist Lehrbeauftragter an der Universität Kassel im Studiengang Global Political Economy. Arbeitsschwerpunkte: Politische Ökonomie, Vergleichende Kapitalismusforschung.

bestef@freenet.de

CHRISTOPH SCHERRER, Dr. habil., ist Professor für Globalisierung und Politik an der Universität Kassel und Geschäftsführender Direktor des International Center for Development and Decent Work. Arbeitsschwerpunkte: Internationale Politische Ökonomie.

@scherrer@uni-kassel.de 